\title{
Dynamic light scattering study of peanut agglutinin: Size, shape and urea denaturation
}

\author{
Sagarika Dev and Avadhesha Surolia \\ Molecular Biophysics Unit, Indian Institute of Science, Bangalore 560 012, India \\ *Corresponding author (Fax, 91-80-23600535; Email, surolia@mbu.iisc.enet.in)
}

\begin{abstract}
Peanut agglutinin (PNA) is a homotetrameric protein with a unique open quaternary structure. PNA shows nontwo state profile in chaotrope induced denaturation. It passes through a monomeric molten globule like state before complete denaturation (Reddy et al 1999). This denaturation profile is associated with the change in hydrodynamic radius of the native protein. Though the molten globule-like state is monomeric in nature it expands in size due to partial denaturation. The size and shape of the native PNA as well as the change in hydrodynamic radius of the protein during denaturation has been studied by dynamic light scattering (DLS). The generation of two species is evident from the profile of hydrodynamic radii. This study also reveals the extent of compactness of the intermediate state.
\end{abstract}

[Dev S and Surolia A 2006 Dynamic light scattering study of peanut agglutinin: Size, shape and urea denaturation; J. Biosci. 31 551-556]

\section{Introduction}

The knowledge of the dimensions of proteins in different state is always essential to get a complete understanding of protein conformation and its role in protein folding. A number of studies have enhanced our understanding of the protein secondary structure in non-native state but less attention has been paid to their dimension during unfolding process (Dev et al 2006b; Mitra et al 2005). Proteins can fold back to their native state by a two-state cooperative process (Sinha et al 2005; Mitra et al 2002; Jackson and Fersht 1991) or in multiple steps populating one or more intermediates (Louzada et al 2003; Hobart et al 2002). However, the intermediates are often transient and difficult to trap for characterizing their thermodynamic and kinetic properties. Often an intermediate called "molten globule" is observed during the folding pathway of many proteins. "Molten globule" state generally possess native like secondary structure but lacks specific tertiary interactions. It is also considerably more compact as compared to the unfolded state (Dolgikh et al 1981; Ohgushi and Wada 1983; Kuwajima 1989; Baum et al 1989; Christensen and Pain 1991; Dobson 1992; Redfield 2004). Direct measurement of any particular quantity in the denatured state is more difficult compared to the native state as the unfolded structure is an ensemble average of several possible conformations. X-ray and neutron scattering techniques (Gast et al 1994) provide precise information on the distance distribution function of the molecule. The dimension of the protein in the different unfolded states gives insight about the protein structural changes that occur during denaturation. As compared to X-ray and neutron scattering the hydrodynamic radius $\left(R_{h}\right)$ measurement is a less direct but an easily measurable physical dimension. $R_{h}$ can be measured by dynamic light scattering (DLS). DLS measures the instantaneous intensity fluctuation arising from the Brownian motion of particles in a small volume. This instantaneous fluctuation can be analysed to get the diffusion coefficients of the particles. $R_{h}$ can be obtained from diffusion coefficient by Stoke's-Einstein relationship. $R_{h}$ obtained this way is the size of a spherical particle that would have a diffusion coefficient equal to the protein in solution. Most proteins are certainly not spherical. Their apparent hydrodynamic radii may strongly vary depending upon their shape and size. Besides, water molecules bound to the proteins may affect the diffusion affecting their hydrodynamic radii which can differs in some situations from the actual size of the molecule. Protein molecules

Keywords. Chaotropic denaturation; DLS; hydrodynamic radius; PNA 


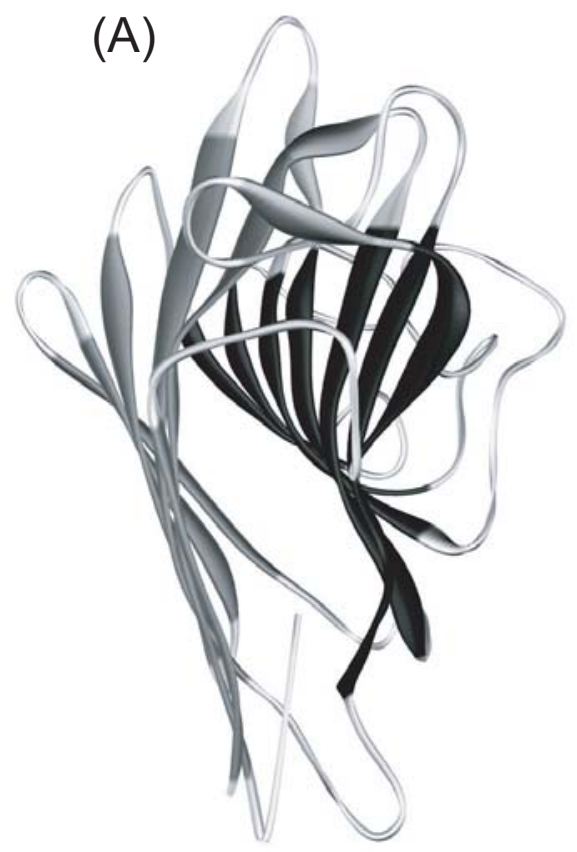

(B)

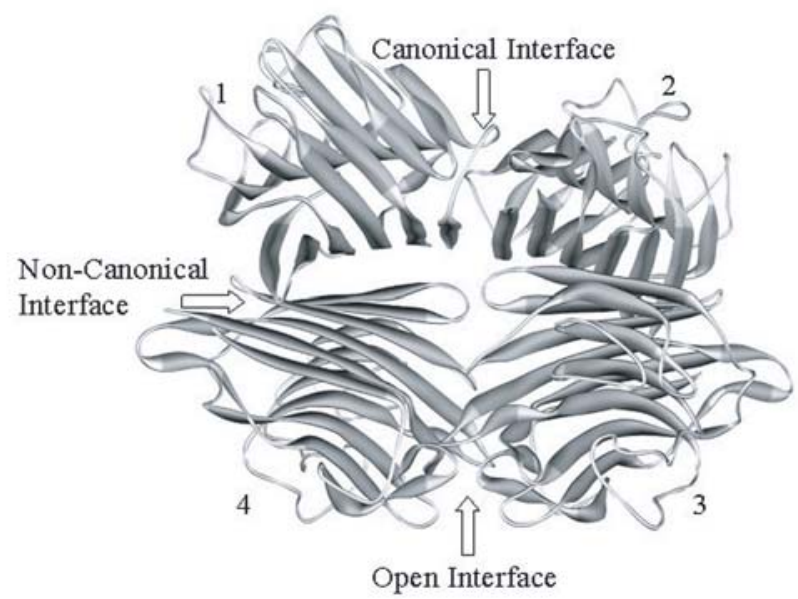

Figure 1. (A) Three-dimensional structural fold of PNA. Six stranded flat back beta sheet (dark gray), seven stranded curved front beta sheet (black) and small five stranded top beta sheet (light gray). (B) Tetrameric PNA with all the three different interfaces marked.

undergo changes in hydrodynamic radii during unfolding. Observation of change in $R_{h}$ may be a good indication of protein unfolding pattern. It has been attempted for a homotetrameric protein peanut agglutinin (PNA) in this study.

PNA is a nonglycosylated homotetrameric protein (Banerjee et al 1996). Mainly three different beta sheets form the monomeric subunit of the tetramer (figure 1A). A flat six stranded back $\beta$ sheet and a seven stranded curved $\beta$ sheet at the front of the molecule are joined by a five stranded short top $\beta$ sheet and several loops. The structure of the monomer is such that three different loops (91-116, 125-135 and 144-148) interact among them and with the front $\beta$ sheet to form a secondary hydrophobic core besides the primary hydrophobic core present between the front and curved $\beta$ sheet. The monomers assemble by three different types of interfaces (figure 1B). Two pairs of monomers (subunit 1-4 and 2-3) associate in a back-to-back fashion with an inclination with respect to each other of $86^{\circ}$ (noncanonical interface, GSIV type). Monomers 1 and 2 involve side by side anti-parallel alignment of the back $\beta$ sheet (canonical). The other interface consists of the monomers 3 and 4 which constitute a unique open interface. PNA shows a non-two state unfolding pattern involving the formation of a molten globule like intermediate (Reddy et al 1999) in thermal and chaotrope induced denaturation. This study is an attempt to follow a chaotrope induced denaturation of PNA by change in $R_{h}$.

\section{Materials and methods}

\subsection{Materials}

Ultra pure urea was purchased from Sigma Chemical Co. (St. Louis, MO, USA). All other reagents used were of the highest purity available. Urea solution was prepared in $20 \mathrm{mM}$ PBS (phosphate buffer saline buffer) with $150 \mathrm{mM} \mathrm{NaCl}$. Each urea solution was prepared fresh and concentration was determined by refractive index measurement as described by Pace (1990).

\subsection{Protein purification}

PNA was extracted from groundnut seeds and purified by lactosyl Sepharose affinity column. Sample purity was checked by SDS-PAGE. The concentration of protein was determined by specific extinction coefficient $A^{1 \%}{ }_{280}=7.7$ (Lotan et al 1975).

\subsection{DLS Measurement}

DLS measurements were done using a DynaPro MS800 equipped with temperature control (Proterion, Protein Solutions, Wyatt Technology, Santa Barbara, CA, USA) operating at wavelength $830 \mathrm{~nm}$ and 256-channel multi tau correlator. The protein solutions were spun at $10 \mathrm{~K}$ 
for $10 \mathrm{~min}$ and filtered through $100 \mathrm{~nm}$ pore size filter membrane (Whatman) into $12 \mu 1$ micro-cell. The translation diffusion coefficient was calculated by Dynamics V6 software, provided by the supplier. Hydrodynamic radius was calculated from translation diffusion coefficient by Stoke's-Einstein relationship. Experiments could not be done at very high urea concentration due to the solvent irregularity. Experiments at the other concentrations of urea did not consider any change in refractive index or viscosity of the solvent. The refractive index and viscosity values were taken for the phosphate buffer solution as provided by the software.

\section{Results and discussion}

PNA unlike several lectins like ECorL, SBA etc; exhibits a non-two state unfolding behaviour. A study on the threestate fitting of the non-two state unfolding induced by urea of PNA to extract all the relevant thermodynamic data has been reported recently (Dev et al 2006a). The study involved fitting of the non-two state unfolding process considering deoligomerisation from the tetramer to the monomeric molten globule initially and then its dissociation and unfolding to completely denatured state. An earlier study from this lab was concerned on the analysis of the sites that become accessible to a protease in the molten globule like intermediate generated during denaturation (Mitra et al 2005). Far and near UV circular dichroism studies in the presence of different concentrations of urea were also presented there.

Generally proteins fold into compact globular domains and are tightly packed. No voids or water molecules are accommodated in the interior though the surface of the protein is rather bumpy. We first determined the size and shape of the native PNA. Volume occupied by a single protein molecule can be found out from equation 1 .

$$
V=1.212\left(\mathrm{~nm}^{3} / \mathrm{Da}\right) * 10^{-3} * M(\mathrm{Da}) .
$$

Here $M$ is the molecular weight in Dalton and $V$, the volume of the protein in $\mathrm{nm}^{3}$. The molecular weight of PNA tetramer was taken as 110,000 $\mathrm{Da}$ (Natchiar et al 2004). The volume of the protein thus obtained was $133.32 \mathrm{~nm}^{3}$. The radius of the smallest globular protein that occupies a volume of $133.32 \mathrm{~nm}^{3}$ was $3.16 \mathrm{~nm}$. This is the theoretically determined value of the radius of the compact PNA molecule.

The hydrodynamic radius is highly affected by the shape of a protein molecule. Generally sedimentation coefficient or frictional coefficient is used to obtain information whether the protein is globular or elongated. Perrin equation, though widely used does not really work for proteins as their surface contours are rather uneven. Therefore, they experience more hydrodynamic drag compared to a smooth ellipsoid. We can have a broad estimation of the shape of the protein by calculating

$$
\frac{f}{f_{\min }}=\frac{S_{\max }}{S} .
$$

$f_{\min }$ or $S_{\max }$ are the frictional coefficient or sedimentation coefficient of an unhydrated sphere corresponding to given mass of a protein. Sedimentation coefficient is related to frictional coefficient by the equation

$$
S=\frac{M(1-v \rho)}{N_{0} f} .
$$

$M=$ molecular weight $(\mathrm{Da}) ; v=$ partial specific volume of the protein (typical value is $0.73 \mathrm{~cm}^{3} / \mathrm{g}$ ); $\rho$ = density of solvent $\left(1.0 \mathrm{~g} / \mathrm{cm}^{3}\right.$ for $\left.\mathrm{H}_{2} \mathrm{O}\right) ; N_{0}$ is the Avogadro's number $\left(6.023 \times 10^{23}\right) ; f$ is the frictional coefficient which depends on the shape of the protein. A more elongated shape creates more hydrodynamic drag and a larger value of $f$. Also, proteins are hydrated - the layer of 'frozen' water held by hydrogen or ionic bonds, or frozen by the interaction at the hydrophobic surface, increases the effective diameter and hydrodynamic drag of protein. Thus the factor $f$ reflects both the shape and hydration.

Again, $f$ is inversely related to diffusion coefficient, which in turn relates to hydrodynamic radius to Stoke'sEinstein relation

$$
D=\frac{k T}{6 \pi \eta R_{h}},
$$

$\eta$ is the viscosity of the solvent $\left(0.01 \mathrm{~g} / \mathrm{cm} . \mathrm{s}\right.$ for $\left.\mathrm{H}_{2} \mathrm{O}\right)$.

By substituting all the relevant parameters solution for $S_{\max }$ is obtained as in eq. (5).

$$
\mathrm{S}_{\max }=0.00361 \times M^{2 / 3} \text {, }
$$

where, $S_{\max }$ is expressed in Svedberg's unit. This gives us the $S_{\max }$ value to be $8.28 S$. The experimentally determined value of $S$ for PNA is $6.4 \mathrm{~S}$ (Fish et al 1978). Thus $S_{\max } / S$ value is found out to be 1.29; which is consistent with a number of globular proteins (Tanfod 1961). The Perrin factor when multiplied by the theoretical radius of PNA gives a value of $4.08 \mathrm{~nm}$. The $R_{h}$ measured by DLS is $3.9 \mathrm{~nm}$ (figure 2). Hence, the theoretical value matches quite well with the experimentally observed value.

DLS has been used to study the hydrodynamic properties of PNA during chaotrope induced denaturation. Several data points have been taken for each urea concentration to calculate the hydrodynamic radii. The data points are marked manually. Data points show a fairly close distribution. The software fits these values to extract out the value of hydrodynamic radius. The fit yields the hydrodynamic radii of each of the species along with their percent $\mathrm{Pd}$ and percent intensity in the regularization view. The values of percent intensity of each species have been plotted in figure 4 . Figure 5 shows a representative regularization fit at 4.3 $\mathrm{M}$ urea concentration. The observed hydrodynamic 


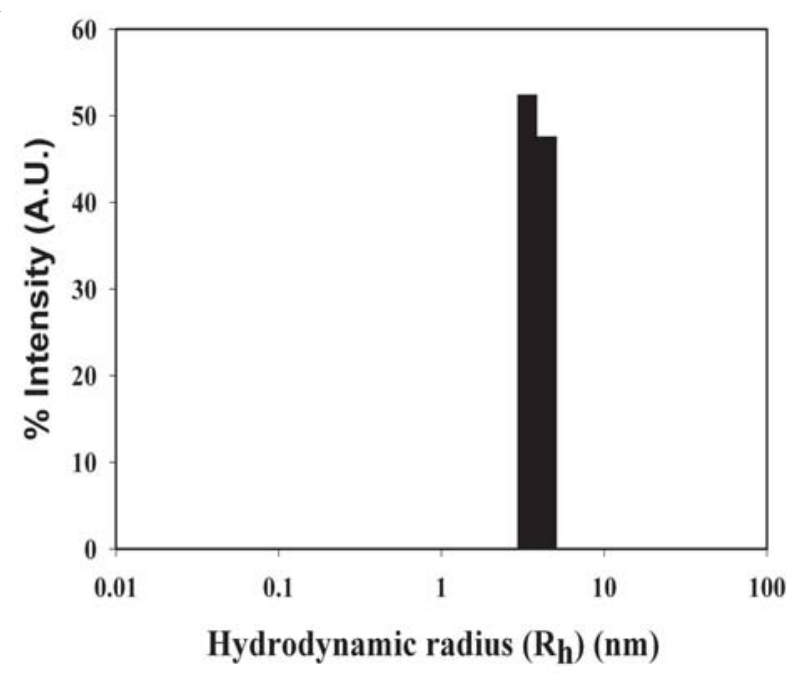

Figure 2. Hydrodynamic radius of native PNA. The protein concentration was $1 \mathrm{mg} / \mathrm{ml}$ in $20 \mathrm{mM}$ PBS with $150 \mathrm{mM} \mathrm{NaCl}$.

Table 1. Hydrodynamic radii and polydispersity of the protein at different concentration of urea.

\begin{tabular}{lcccc}
\hline $\begin{array}{l}\text { Urea } \\
\text { Conc. }(M)\end{array}$ & $R_{h}(1)(\mathrm{nm})$ & $P d(\%)$ & $\begin{array}{c}R_{h}(2) \\
(\mathrm{nm})\end{array}$ & $P d(\%)$ \\
\hline 0 & 3.9 & 18.4 & & \\
1.07 & 4.1 & 12.8 & & \\
2.15 & 4.2 & 13.8 & 11.7 & 15.9 \\
2.69 & 5 & 22.1 & 14.7 & 26.3 \\
3.2 & 6.2 & 19.8 & 18.1 & 24 \\
3.76 & 8.7 & 22.6 & 22.3 & 23.1 \\
4.3 & 10.7 & 24.6 & 23.7 & 13.9 \\
4.84 & 8 & 13.2 & 21.6 & 11.5 \\
5.3 & 7.6 & 14 & 20 & 7.5 \\
6.4 & 8.3 & 11.3 & 20.9 & 7.5 \\
7.5 & 6.7 & 0.0 & 20.7 & 14.8 \\
8.6 & 6 & 13.2 & 20.5 & 0.0 \\
\hline
\end{tabular}

radii are shown in table 1 . The hydrodynamic radii can not be followed at very high urea concentration due to solvent irregularity. A perusal of the profile of the hydrodynamic radii (figure 3) shows generation of intermediate species along with denatured state from $2 \mathrm{M}$ urea onwards and the protein radius becomes almost constant at $5 \mathrm{M}$ urea.

The intensity profile reported here (figure 4 ) shows the generation of the two species more precisely. The percent intensity does not correspond to the concentration of each species present; rather it is an indicator of relative population of the species. Initially at very low urea concentration only native form is present. With increase in concentration both the species start forming and as denatured species has higher

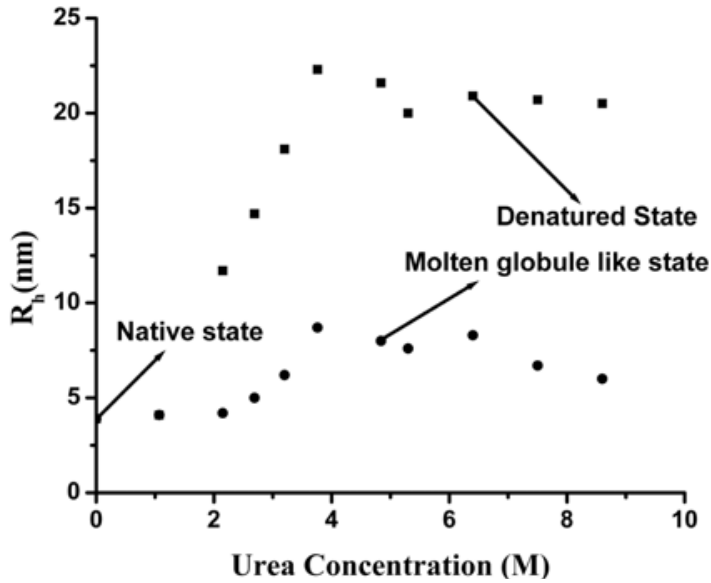

Figure 3. Change in hydrodynamic radius of the native PNA with increasing concentration of urea. Generation of the molten globule like state along with the denatured state can be identified. The value of hydrodynamic radius has been taken by deleting the odd data points and taking the value from the regularization view of the software.

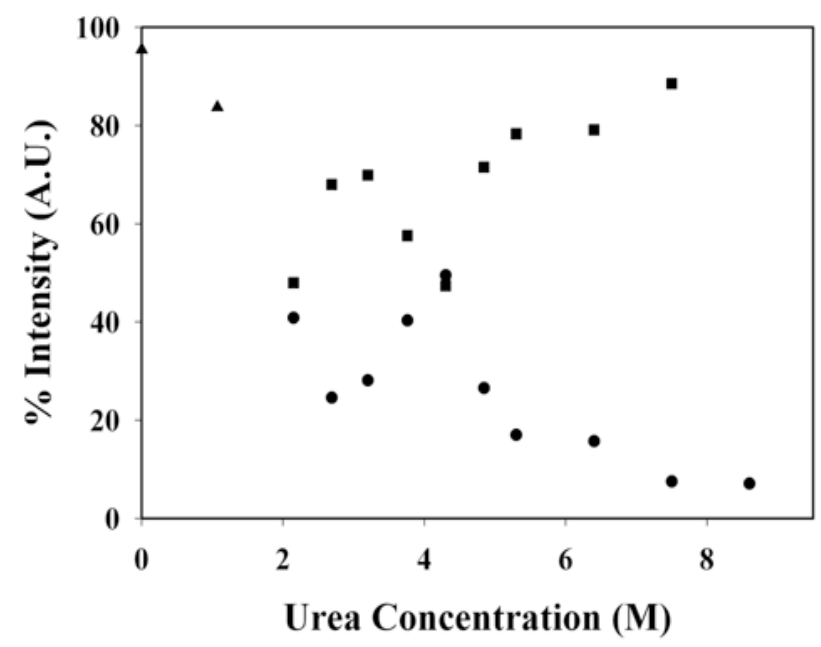

Figure 4. Intensity profile of all the species generated at different concentration of urea. The value of percent intensity is shown along with the hydrodynamic radius obtained by the regularization fit of the DLS data according to the software provided by the manufacturer. Those values have been taken and plotted. $(\boldsymbol{\Delta})$, the native tetramer, $(\boldsymbol{\bullet})$, denatured species and $(\bullet)$, molten globule like state.

hydrodynamic radius compared to the intermediate species, denatured species contributes more in total intensity than the molten globule like state. With increase in concentration of urea as more and more molten globule like state starts forming, percent intensity of the denatured state starts decreasing and reaches the minima at $4.3 \mathrm{M}$ urea. At the 


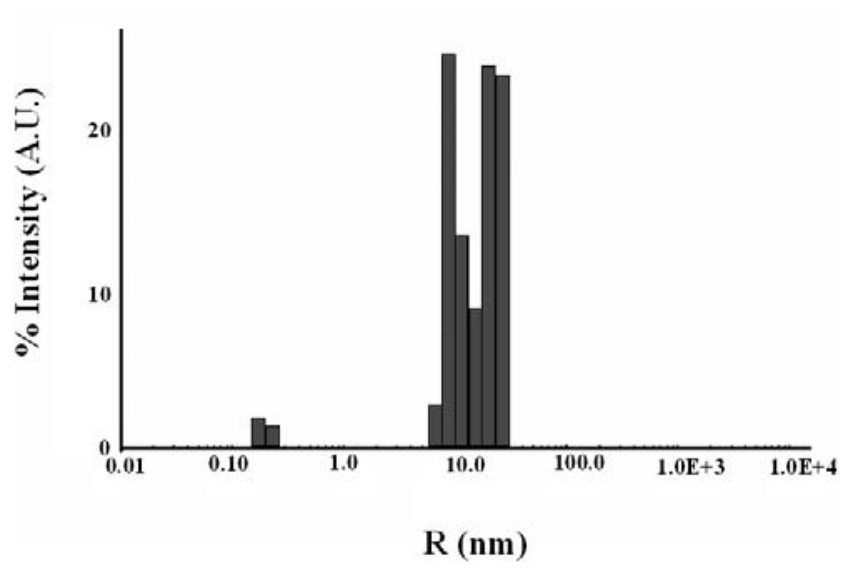

Figure 5. A representative regularization fit at $4.3 \mathrm{M}$ urea concentration. The fit is obtained by the regularization fit, obtained as described in $\S 3$, of the experimental correlation function. The fit shows the presence of three species. The first peak is from the solvent. Any species having radius less than $1 \mathrm{~nm}$ is considered to be emanating from the solvent. The hydrodynamic radii for the other two species are $10.7 \mathrm{~nm}$ and $23.7 \mathrm{~nm}$. These values have been taken for comparison at different urea concentrations.

same time the contribution to the total intensity count by the intermediate species increases. After $4.3 \mathrm{M}$ of urea concentration, molten globule, like intermediate transforms to denatured species, hence its percent intensity starts decreasing. Analysis of the hydrodynamic radius $\left(R_{h}\right)$, as presented here, is a good way to follow the simultaneous generation of two species. Fluorescence and circular dichroism in contrast provide information about the species present in the solution as a whole and constitute an average measurement of their physical properties in an ensemble but $R_{h}$ on the other hand deals with the individual property. The compactness factor of the molten globule like state can be described as:

$$
\text { Compactness Factor }=\frac{R_{D}-R_{I}}{R_{D}-R_{N}},
$$

$R_{D}, R_{I}$ and $R_{N}$ are the hydrodynamic radius of denatured, intermediate and native state respectively. If $R_{D}, R_{I}$ and $R_{N}$ are taken to be $20.5 \mathrm{~nm}, 10.7 \mathrm{~nm}$ and $3.9 \mathrm{~nm}$ respectively then compactness factor comes out to be $59.03 \%$. Almost $40 \%$ unfolding of molten globule like state tallies very well with the amount present in the isothermal melting study with urea (Dev S, Nirmala Devi K, Sinha S and Surolia A, unpublished result). The homogeneity of the solution is reflected in the parameter percent polydispersity (Pd). Polydispersity is the measurement of standard deviation of the size of the particle. Native PNA shows polydispersity around $15-20 \%$, corresponding to a monomodal species. With increase in concentration of urea polydispersity keeps on increasing to more than $25 \%$ (table 1) along with the generation of the molten globule like state and the denatured state. Polydispersity shows a maximum value at $4.3 \mathrm{M}$ urea concentration. With further increase in concentration of urea the concentration of the molten globule like state decreases in solution and the denatured species predominates. Polydispersity also decreases reflecting increase in homogeneity of the solution. Native PNA always exists with a small percentage of aggregate but there is no evidence of increase in percentage of aggregate compared to the native state during denaturation.

\section{Refolding}

Hydrodynamic radii during refolding of PNA at $7 \mathrm{M}$ urea concentration was also followed by DLS. Dilution of the denatured protein from $7 \mathrm{M}$ urea causes aggregation. Refolding experiments were not successful due to fluctuation in intensity due to aggregation. A preliminary study on refolding of PNA by tryptophan fluorescence shows that it passes through an intermediate state. The first step, folding from denatured to the intermediate step is quite fast while the second step viz. the folding from the intermediate state to the native state is very slow and requires several hours of dialysis (Dev et al, 2006a).

\section{Conclusion}

DLS has been used to monitor change in dimension of the protein during denaturation and renaturation (Nicholi and Benedek 1976; Gast et al 1986). This has been extended here for studying chemical denaturation of PNA. PNA, the tetrameric protein exhibits scattering behaviour which is consistent with a globular protein as supported from the DLS studies. The change in $R_{h}$ during chemical denaturation is a good measure of protein radii during unfolding. It provides a direct evidence for the generation of an intermediate during the unfolding process of PNA as exemplified here.

\section{Acknowledgements}

The work is supported by the grant of the Department of Biotechnology, New Delhi. We thank Department of Science and Technology, New Delhi for the DLS instrument under the scheme "Fund for Improvement of S\&T Infrastructure (FIST)".

\section{References}

Banerjee R, Das K, Ravishankar R, Suguna K and Surolia A 1996 Conformation, protein-carbohydrate interactions and a novel 
subunit association in the refined structure of Peanut lectinlactose complex; J. Mol. Biol. 259 281-296

Baum J, Dobson C M, Evans P A and Hanley C 1989 Characterization of a partly folded protein by NMR methods: studies on the molten globule state of guinea pig alphalactalbumin.; Biochemistry 28 7-13

Christensen H and Pain R H 1991 Molten globule intermediates and protein folding; Eur. Biophys. J. 19 221-229

Dev S, Nirmala Devi K, Sinha S and Surolia A 2006a Thermodynamic analysis of three state denaturation of peanut agglutinin; IUBMB Life 58 549-555

Dev S, Khan R H and Surolia A 2006b 2, 2, 2-Trifluoroethanol induced structural change of Peanut agglutinin at different $\mathrm{pH}$ : A comparative account; IUBMB Life 58 473-479

Dobson C M 1992 Resting places on folding pathways; Curr. Opin. Struct. Biol. 2 6-12

Dolgikh D A, Gilmanshin R I, Brazhnikov E V, Bychkova V E, Semisotnov G V, Venyaminov S Y and Ptitsyn O B 1981 AlphaLactalbumin: compact state with fluctuating tertiary structure?; FEBS Lett. 136 311-315

Fish W W, Hamlin L M and Miller R L 1978 The macromolecular properties of peanut agglutinin; Arch. Biochem. Biophys. 190 693-698

Gast K, Damaschun H, Misselwitz R, Muller-Frohne M, Zirwer D and Damaschun G 1994 Compactness of protein molten globules: temperature-induced structural changes of the apomyoglobin folding intermediate; Eur. Biophys. J. 23 297-305

Gast K, Zirwar D, Welfle H, Bychkova V E and Ptitsyn O B 1986 Quasi-elastic light scattering from human alpha-lactalbumin: comparison of molecular dimensions in native and molten globule states; Int. J. Biol. Macromol. 83 231-236

Hobart S A, Meinhold D W, Osuna R and Colon W 2002 From two-state to three-state: The effect of the P61A mutation on the dynamics and stability of the factor for inversion stimulation results in an altered equilibrium denaturation mechanism; Biochemistry 41 13744-13754

Jackson S E and Fersht A R 1991 Folding of Chymotrypsin Inhibitor 2.1.Evidence for a two-state transition; Biochemistry 30 10428-10435
Kuwajima K 1989 The molten globule state as a clue for understanding the folding and cooperativity of globular-protein structure; Proteins Struct. Funct. Genet. 6 87-103

Lotan R, Skutelsky E, Danon D and Sharon N 1975 The purification, composition, and specificity of the anti-T lectin from peanut (Arachis hypogaea); J. Biol. Chem. 250 8518-8523

Louzada P R, Sebollela A, Scaramello M E and Ferreira St T 2003 Predissociated dimmers and molten globule monomers in the equilibrium of Yeast Glutathione Reductase; Biophys. J. 85 3255-3261

Mitra N, Sinha S, Kini R M and Surolia A 2005 Analysis of Peanut agglutinin molten globule-like intermediate by limited proteolysis; Biochim. Biophys. Acta 1725 283-289

Mitra N, Srinivas V R, Ramya T N C, Ahmad N, Reddy G B and Surolia A 2002 Conformational stability of legume lectins reflect their different modes of quaternary association: solvent denaturation studies on concanavalin A and winged bean acidic agglutinin; Biochemistry 41 9256-9263

Natchiar S K, Jeyaprakash A A, Ramya T N C, Thomas C J, Suguna K, Surolia A and Vijayan M 2004 Structural plasticity of peanut lectin:an X-ray analysis involving variation in $\mathrm{pH}$, ligand binding and crystal structure; Acta. Crystalogr. D60 211-219

Nicoli D F and Benedek G B 1976 Study of thermal denaturation of lysozyme and other globular proteins by light scattering spectroscopy; Biopolymers 15 2421-2437

Ohgushi M and Wada A 1983 "Molten-globule state": a compact form of globular proteins with mobile side-chains; FEBS Lett. $16421-24$

Pace C N 1990 Conformational stability of globular proteins; Trends Biochem. Sci. 15 14-17

Reddy G B, Srinivas V R, Ahmed N and Surolia A 1999 Molten globule-like state of Peanut lectin monomer retains its carbohydrate specificity; J. Biol. Chem. 274 4500-4503

Redfield C 2004 Using magnetic resonance spectroscopy to study molten globule states of proteins; Methods 34 121-132

Sinha S, Mitra N, Kumar G, Bajaj K and Surolia A 2005 Unfolding studies on soybean agglutinin and concanavalin a tetramers: a comparative account; Biophys. J. 88 1300-1310

Tanford C 1961 Physical chemistry of macromolecules (New York: John Wiley N) pp 358

MS received 6 June 2006; accepted 26 September 2006

ePublication: 3 November 2006

Corresponding editor: VidyanAND NANJUndiaH 